\title{
Improved Null Steering with Sidelobe Canceller for Linear Antenna Arrays
}

\author{
Sharlaine Nicole Monteiro ${ }^{1}$, H.G.Virani ${ }^{2}$ \\ Electronics and Telecommunication Engg., Goa College of Engineering, Farmagudi-Goa, India ${ }^{1}$ \\ Professor and Head, Electronics and Telecommunication Engg., Goa College of Engineering, Farmagudi-Goa, India ${ }^{2}$
}

\begin{abstract}
Reduction of interference is one of the major tasks in a communication system. Jamming sources and clutter are the two major sources of interferences. The interferers are generally estimated by Direction of arrival (DOA) techniques and then they are suppressed by introducing nulls in those directions. Suppression of interference leads to higher Signal to Noise ratio and superior data rates. Clutter enters through the sidelobes. Therefore sidelobe reduction reduces the effect of clutter. Null Steering Algorithm generally estimate weights which are then multiplied with weights generated for sidelobe suppression. This paper focuses on introduction and steering of nulls in any desired direction and sidelobe suppression.
\end{abstract}

Keywords: Null Steering; Array Factor; Adaptive Beamforming; Antenna Array; Modified Bartlett- Hanning window.

\section{INTRODUCTION}

Beamforming or spatial filtering is a signal processing technique used in sensor arrays for directional signal transmission or reception. Beamforming accentuates or attenuates signals that arrive from specific directions and helps to distinguish between signals of interest and interfering signals from other directions.

Conventional beamformers use filter weights chosen independent of data received. They thus do not account for any interference and thus susceptible to interference signals. On the other hand Adaptive Beamformers account for interference signals. An adaptive beamformer algorithm chooses the filter weights based on received data. The nulls in the radiation pattern are then placed at angles corresponding to the interference signals. Beam forming where nulls are placed in the directions of interferences is known as null steering.document is a template. An electronic copy can bedownloaded from the conference website. For questions on paper guidelines, please contact the conference publicationscommittee as indicated on the conference website. Information about final paper submission is available from the conference website.

\section{A. Null steering}

Null steering uses the directions of sources toward which nulls are desired to be placed for estimating the weights on each element.Various Adaptive Beamforming and Null steering algorithms have been developed which include controlling the antenna array excitation amplitude-only, the excitation phase only, the element position only and thecomplex weights (both the amplitude and the phase). The control of both amplitude and phase i.e. the use of complex weights of each array element produces the closest pattern to the desired radiation pattern because it has greater degrees of freedom [5]. Some of the algorithms used for null steering by using complex weights are the Generic algorithm (GA) and the Particle Swarm Optimization (PSO). According to [1], in these algorithms if the position of a single null is required to be changed, the whole set of weights has to be recomputed. For large arrays this is consumes a lot of time. Reference [1] suggested the use of Independent weight control, wherein only the weights corresponding to the changed null have to be recalculated and modified and the other weights are not affected.

The techniques to remove interference signals do not give the desired reduction in the side lobe magnitude and can also cause shifting of the mainlobe. Low side lobe beams benefit clutter suppression techniques and require fewer beamforming sensors to reduce side lobe jamming.

\section{B. Sidelobe Suppression}

The techniques for null steering do not provide thedesired reduction in the sidelobe magnitude. Sidelobe suppression can cause shifting and broadening of the mainlobe. Sidelobe suppression in arrays also leads to an increase in the number of elements of the array for the same number of nulls as compared to a system without sidelobe suppression. However low sidelobe beams aid in clutter suppression techniques and require fewer beamforming sensors to reduce sidelobe jamming.

\section{INDEPENDENT WEIGHT CONTROL}

\section{A. Array Factor}

Consider an array of $\mathrm{N}$ elements with equal spacing $\mathrm{d}$. All elements are placed in a straight line with first element considered as reference point as shown in Fig. 1. Angle of arrival is considered $\theta$. Consider that the current amplitudes of all elements in the linear array are uniform and $\alpha$ is considered as the progressive phase. The array factor is given as:

$$
\begin{aligned}
A F= & \sum_{i=1}^{N} e^{j(i-1) \psi} \\
& =\mathrm{kd} * \cos \theta+\alpha \\
& k=2 \Pi / \lambda \text { is the wave number. }
\end{aligned}
$$




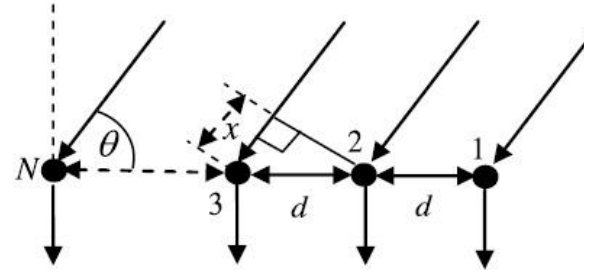

Fig.1. Uniform Linear Array of $\mathrm{N}$ elements

The general formula for first stage outputs of an $\mathrm{N}$ element array is given by:

$y_{1, j}=z^{j-1}\left(z-z_{1}\right) \quad$ for $\mathrm{j}=1,2, \ldots, N-1$

The general formula for second stage outputs of an $\mathrm{N}$ element array is given by

$\left.\mathrm{y}_{2, \mathrm{j}}=\mathrm{z}^{\mathrm{j}-1}\left(\mathrm{z}-\mathrm{z}_{1}\right) \mathrm{z}-\mathrm{z}_{2}\right) \quad$ for $\mathrm{j}=1,2, \ldots, \mathrm{N}-1$

Thus a general formula for output of each stage for an $\mathrm{N}$ element array is given by

$\left.\mathrm{y}_{\mathrm{i}, \mathrm{j}}=\mathrm{z}^{\mathrm{j}-1}\left(\mathrm{z}-\mathrm{z}_{1}\right) \mathrm{z}-\mathrm{z}_{2}\right) \ldots\left(\mathrm{z}-\mathrm{z}_{\mathrm{k}}\right)$

If the beam is to be steered in the desired direction $\theta$ s, the progressive phase shift is given by:

$\alpha=-k d * \cos \theta_{\mathrm{s}}$

In expanded form the array factor is:

$$
\mathrm{AF}=1+\mathrm{e}^{\mathrm{j}}+\mathrm{e}^{2 \mathrm{j}}+\ldots+\mathrm{e}^{\mathrm{j}(\mathrm{N}-1) \psi}
$$

Let $\mathrm{e}^{\mathrm{j}}=\mathrm{z}$, the array factor can now be expressed as

$$
\mathrm{AF}=1+\mathrm{z}+\mathrm{z}^{2}+\ldots+\mathrm{z}^{(\mathrm{N}-1)}
$$

Hence, we get a polynomial of order (N-1). Polynomial of the array factor has $(\mathrm{N}-1)$ roots on the unit circle in the complex plane. Zeros of the above polynomial has fixed positions along the unit circle. These positions are $\mathrm{z}_{1}, \mathrm{z}_{2}, \ldots$ . , $\mathrm{z}_{\mathrm{N}-1}$. Polynomial of the array factor can be expressed in factorized form as:

$$
\mathrm{AF}=\left(\mathrm{z}-\mathrm{z}_{1}\right)\left(\mathrm{z}-\mathrm{z}_{2}\right) \ldots\left(\mathrm{z}-\mathrm{Z}_{\mathrm{N}-1}\right)
$$

The roots of the polynomial $\mathrm{z}_{1}, \mathrm{z}_{2}, \ldots, \mathrm{z}_{\mathrm{N}-1}$ corresponds to the nulls of the array factor in the fixed directions $\theta_{1}, \theta_{2}, \ldots$ ., $\theta_{\mathrm{N}-1}$ respectively.

In order to get zeros of the polynomial of the array factor on the unit circle of the complex plane in arbitrary directions, the coefficients of the polynomial should be changed accordingly [6]. To steer nulls in the arbitrary directions, weights of the array elements must be updated. Therefore, array factor can be expressed in the following way for the array of $\mathrm{N}$ elements:

$\mathrm{AF}=\mathrm{A}_{0}+\mathrm{A}_{1} \mathrm{z}+\mathrm{A}_{2} \mathrm{z}^{2}+\ldots+\mathrm{A}_{\mathrm{N}-1} \mathrm{z}^{(\mathrm{N}-1)}$

\section{B. Independent Weight Control by using Complex} Weights

The structure as proposed by [1] for independent null steering is as shown in Fig. 2.

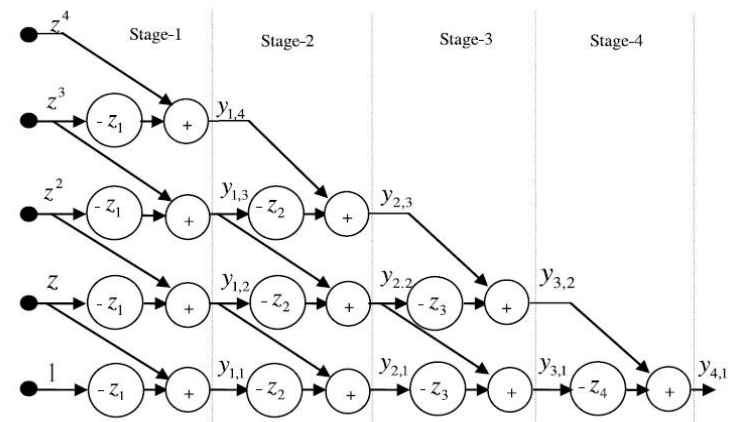

Fig. 2. Independent null steering structure for uniform linear array of five elements

Each output of the first stage is denoted by $y_{1, j}$, for $j=1,2$, . ., N-1. So $\mathrm{y}_{1,1}=\mathrm{z}-\mathrm{z}_{1}, \mathrm{y}_{1,2}=\mathrm{z}\left(\mathrm{z}-\mathrm{z}_{1}\right), \mathrm{y}_{1,3}=\mathrm{z}_{2}\left(\mathrm{z}-\mathrm{z}_{1}\right), \mathrm{y}_{1,4}=$ $\mathrm{z}_{3}\left(\mathrm{z}-\mathrm{z}_{1}\right)$

with $\mathrm{i}=1, \ldots, \mathrm{N}-1 ; \mathrm{j}=1, \ldots, \mathrm{N}-\mathrm{i} ; \mathrm{k}=1, \ldots, \mathrm{i}$. This array will have $(\mathrm{N}-1)$ stages.

The final output for the array will be

$$
\left.\mathrm{y}_{\mathrm{N}-1,1}=\left(\mathrm{z}-\mathrm{z}_{1}\right) \mathrm{z}-\mathrm{z}_{2}\right) \ldots\left(\mathrm{z}-\mathrm{z}_{\mathrm{n}-1}\right)
$$

which thus gives the array factor.

The relation between the direction of the $i^{\text {th }}$ null $\theta_{i}$ and the corresponding weight $\mathrm{z}_{\mathrm{i}}$ is given as

$$
\mathrm{z}_{\mathrm{i}}=\exp \left(\mathrm{j}\left(\alpha+\mathrm{kd} \mathrm{d}^{*} \cos \theta_{\mathrm{i}}\right)\right)
$$

\section{Sidelobe Suppression}

We use the coefficients of an L+2 length Modified Bartlett -Hanning window to suppress the sidelobes. This however requires the array size to be increased by L-1 elements. Like the Bartlett, Hanning, and Hamming windows, this window has the mainlobe at the origin and asymptotically decaying sidelobes on both sides. It is a linear combination of weighted Bartlett and Hanning windows with near sidelobes lower than both Bartlett and Hanning and with far sidelobes lower than both Bartlett and Hamming windows. The mainlobe width of the modified BartlettHanning window is not increased relative to either Bartlett or Hanning window mainlobes. The spectrum of the modified Bartlett-Hanning Window is shown in Fig. 3.

The coefficients of the Modified Bartlett-Hanning window are computed from the following equation:

$w(n)=0.62-0.48\left|\left(\frac{n}{N}-0.5\right)\right|+0.38 \cos \left(2 \Pi\left(\frac{n}{N}-0.5\right)\right)$

with $0 \leq \mathrm{n} \leq \mathrm{N}$ and window length is $(\mathrm{N}+1)$.

The array factor obtained using these coefficients is multiplied with the array factor obtained using the Independent weight control algorithm to give the final array factor and thus the final weights to be applied to the antenna array to get the desired pattern.

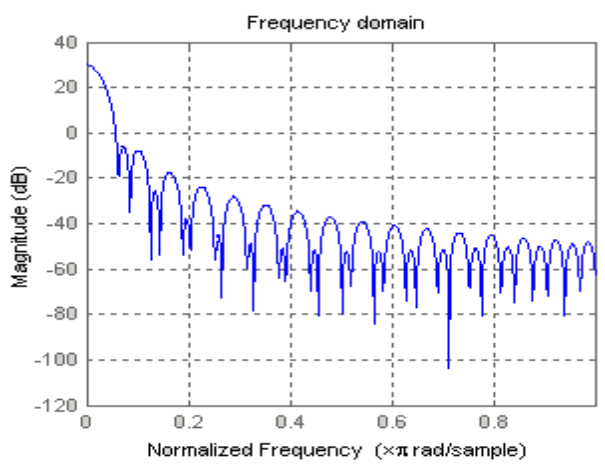

Fig. 3. Spectrum of Bartlett Hanning Window 


\section{III.SIMULATION AND RESULTS}

The block diagram for the system setup is shown in Fig. 4. The signal received from the antenna is analysed in the block 'Adaptive system to control weights'. Using the MUSIC algorithm the interferer directions are estimated. These estimations are then used by the Independent weight null steering algorithm to generate weights to steer the nulls. An array factor is formed using these weights.

Weights are separately generated for sidelobe suppression using the coefficients of the Modified Bartlett-Hanning window. And a second array factor equation is formed. Both the array factors are multiplied to give the final set of weights. These weights are then applied back at the array input. This will result in an output array pattern with nulls in the direction of the interferers and reduced sidelobes.

The simulation was carried out in MATLAB for a uniform linear array with a separation of $15 \mathrm{~cm}$ between the array elements.

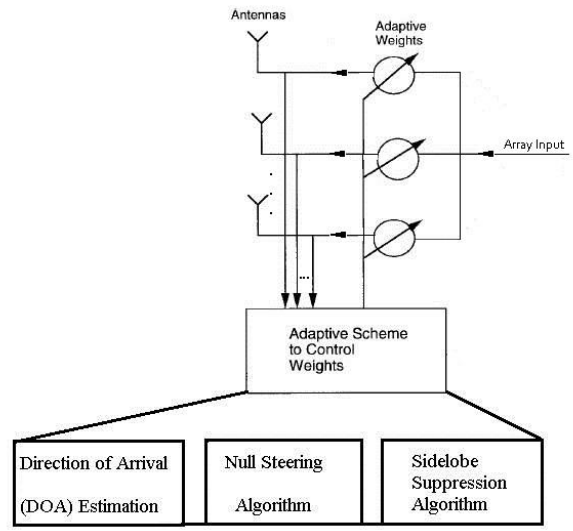

Fig. 4. Block Diagram of System

The frequency range of operation was 900-1100MHz. System was simulated by introducing random AWGN noise and interferences located at $\left[\begin{array}{llll}30^{\circ} & 45^{\circ} & 70^{\circ} & 125^{\circ}\end{array}\right.$ $160^{\circ}$ ].Simulation can be performed for different angles of interferences.

Initially the results of a six element array pattern are shown. It can generate nulls in the five directions of interferences.

The Direction of Arrival Estimation using MUSIC algorithm estimates the interferences angles as shown in Fig. 5[8].

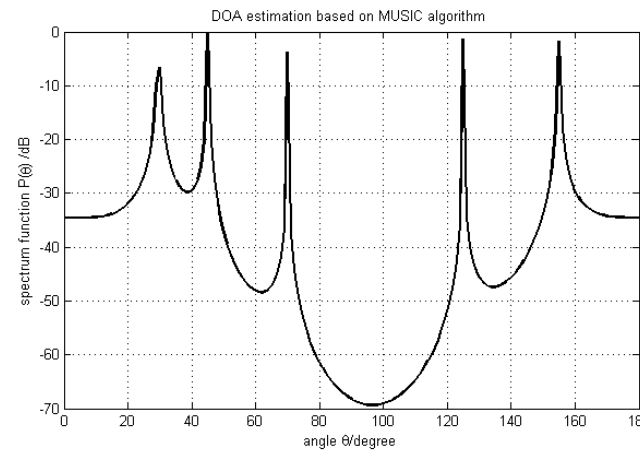

Fig. 5. Direction of Arrival Estimation
Using Independent weight control algorithm, the corresponding weights obtained were $z_{1}=-0.9127+$ $0.4086 \mathrm{i}, \mathrm{z}_{2}=-0.6057+0.7957 \mathrm{i}, \mathrm{z}_{3}=0.4762+0.8793 \mathrm{i}$, $\mathrm{z}_{4}=-0.2291-0.9734 \mathrm{i}$ and $\mathrm{z}_{5}=-0.9570$ 0.2901 irespectively.

Fig. 6 shows the array pattern of the array using Independent weight control with complex weights method of Null steering. The dashed line array pattern is the output when a Conventional Beamformer is used without any null steering.

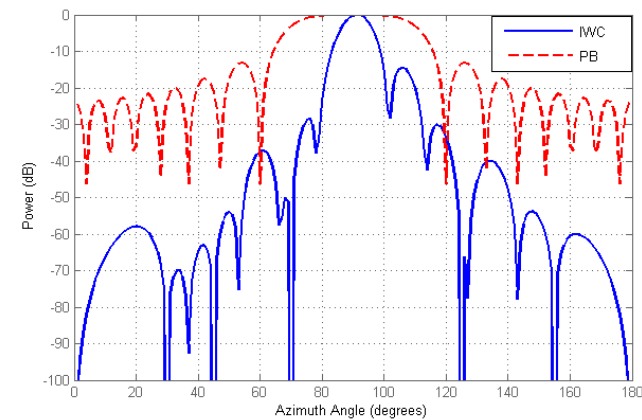

Fig. 6. Independent weight control with complex weight (IWC- Independent Weight Control: PB- Phased Beamformer)

Fig. 7 shows the array pattern of an extended fifteen element array which generates five nulls along with sideband suppression using the coefficients generated using theModified Bartlett-Hanning Window of length 12. The sidelobe suppression obtained is around $29 \mathrm{~dB}$.

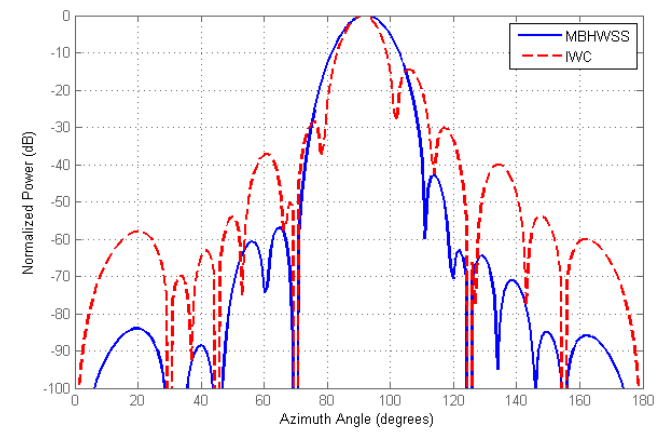

Fig. 7. Sidelobe Suppression Using Modified Bartlett-Hanning Window

(MBHWSS- Modified Barlett-Hanning Window Sidelobe Suppression; IWC- Independent Weight Control)

\section{IV.CONCLUSION}

Simulation was performed for a system containing a linear antenna array. The direction of arrival of the interferers wasestimated. The Independent Weight Control Null steering algorithm was applied on the array to generate weights such that nulls were introduced in the direction of the interferers. Sidelobe Suppression was applied using coefficients of the Modified Bartlett-Hanning Window. With sidelobe suppression we got a sidelobe level of $43 \mathrm{~dB}$ which is around $29 \mathrm{~dB}$ below the result without sidelobe suppression 


\section{REFERENCES}

[1] Z.U.Khan, A.Naveed, I.M.Qureshi, F.Zaman, "Independent null steering by decoupling complex weights," IEICE Electronics Express, vol.8, no.13, pp.-1008-1013, 10 July 2011.

[2] Shahid Mehmood, Zafar Ullah Khan, Fawad Zaman and Bilal Shoaib, "Performance analysis of the different null steering techniques in the field of adaptive beamforming," Research Journal of Applied Sciences, Engineering and Technology 5(15): 4006-4012, 2013

[3] H. M.Ibrahim, "Null steering by real-weight control- a method of decoupling the weights," IEEE Trans. Antennas and Propagation, Vol. 39, No. 11, Nov.1991.

[4] Lal C. Godara, "Applications of antenna arrays to mobile communications, Part I: Performance Improvement, Feasibility, and System Considerations," Proceedings of the IEEE, Vol.85. No. 7, July 1997.

[5] K. Guney, A.Akdagli, "Null steering of linear antenna arrays using modified TABU search algorithm,"Progress in Electromagnetics Research, PIER, vol.33, pp. 167-182, 2001.

[6] Karim, H. and M. Viberg, 1996 "Two decades of array signal processing research: The parametric approach," IEEE Signal Proc. Mag., 13(4): 67-94.

[7] S.A. Schelkuno_, "A mathematical theory of linear arrays," Bell Syst. Tech.J., vol. 22, pp. 80-107, 1943.

[8] Honghao Tang, "DOA estimation based on MUSIC algorithm,'Institutionen För Fysik och Elektroteknik.

[9] Md Abdus Samad, "A novel window function yeilding suppressed mainlobe width and minimum sidelobe peak", International Journal of Computer Science, Engineering and Information Technology(IJCSEIT), Vol 2, No.2, April2012. 